\title{
CONTINUOUS-TIME METHODS IN THE STUDY OF DISCRETELY SAMPLED FUNCTIONALS OF LÉVY PROCESSES. I. THE POSITIVE PROCESS CASE
}

\author{
MICHAEL SCHRÖDER *
}

\begin{abstract}
In this paper we develop a constructive approach to studying continuously and discretely sampled functionals of Lévy processes. Estimates for the rate of convergence of the discretely sampled functionals to the continuously sampled functionals are derived, reducing the study of the latter to that of the former. Laguerre reduction series for the discretely sampled functionals are developed, reducing their study to that of the moment generating function of the pertinent Lévy processes and to that of the moments of these processes in particular. The results are applied to questions of contingent claim valuation, such as the explicit valuation of Asian options, and illustrated in the case of generalized inverse Gaussian Lévy processes.
\end{abstract}

Keywords: Lévy process; orthogonal polynomial; constructive method for functionals of Lévy processes; Laguerre reduction series; generalized inverse Gaussian Lévy process

2000 Mathematics Subject Classification: Primary 60G51; 91B28; 65C50

Secondary $33 \mathrm{C} 45 ; 33 \mathrm{C} 90$

\section{Introduction}

In this paper we demonstrate a constructive approach to the study of functionals of Lévy processes the basic idea of which is to use continuous-time methods to study discrete-time situations, and vice versa. The one notion we concentrate on is that of discretely sampled functionals, a concept made precise in Section 4. Such fundamentals include, as a simple but typical example, expectations of the form

$$
\mathrm{E}[\rho(\Sigma)], \quad \text { where } \Sigma=\sum_{u \in \mathcal{T}} X_{u},
$$

the latter obtained as the sum of finitely many random variables $X_{u}$. Taking $X$ as a process, these discretely sampled functionals can be understood as discrete-time approximations to the second notion we concentrate on: the continuously sampled functionals given by the expectations

$$
\mathrm{E}\left[\rho\left(A_{t, T}(X)\right)\right], \quad \text { where } A_{t, T}(X)=\int_{t}^{T} X_{u} \mathrm{~d} u .
$$

Examples of discretely and continuously sampled functionals are common and occur in areas as diverse as the physics of random media and finance or insurance. In Section 4 we discuss applications to these last two areas which include the valuation of Asian options. This example shows that, apart from a structure theory, one wants representations for these functionals which can be used for numerical work as well.

Received 19 September 2005; revision received 29 September 2006.

* Postal address: Keplerstrasse 30, D-69469 Weinheim (Bergstrasse), Germany. 
Our main contribution in this paper is the development of an approach which addresses both aspects, giving a structure theory for the above functionals and also furnishing implementable formulae for their computation. Assuming that $X$ is a Lévy process, the approach is in terms of the characteristic function of $X$ and derived concepts such as the moments of $X$. Assuming additionally that $X$ is positive, it proceeds in three principal steps as follows.

As a first step we develop a method for coping with the principal difficulty in studying the continuously sampled functionals: integrating with respect to time destroys the law of the original process. The idea is to study these functionals as limits of discretely sampled ones. The key difficulty then is to give estimates for the rate of this convergence, and this is addressed in Section 5. There we show how, under differentiability assumptions on the functions $\rho$, the continuously sampled functionals differ from the corresponding discretely sampled ones by an error proportional to the reciprocal of the number of sample points.

A reduction thus occurs to the study of the discretely sampled functionals alone. Here the principal difficulty comes from the dimensionality of the problems. Indeed, if the random variables $X_{u}$, for $u \in \mathcal{T}$, are independent, each of them adds one dimension to the expectation: sampling over a three-month period thus results in 60-dimensional integrals. Dependencies among the $X_{u}$ will introduce additional relations into these integrals and, thus, additional difficulties in their computation. As a second step, in Section 6 we therefore develop a normalization of discretely sampled functionals according to which they are constructed in terms of independent random variables.

As a third step, in Section 8 we develop Laguerre reduction series for the step-two functionals. With the pertinent results assembled in Section 3, this development proceeds along the lines of Schröder (2005a), as follows: expand the probability densities of the above random variables $\Sigma$ into Laguerre polynomials and try to take the expectation through the resulting summation. We find that the validity of these operations can be characterized in terms of the characteristic function of $X$ and its asymptotic behaviour, and that the resulting reduction series are indeed in terms of the moments of $X$. From the point of view of the general framework developed in Schröder (2006a), the approach furnished is the one complementary to that of Dufresne (2000), and yields reduction series particularly apt for computations.

We therefore use these results to develop an explicit valuation of Asian-type options in Section 9, and illustrate them for generalized inverse Gaussian Lévy processes $X$, as reviewed in Section 2. With Schröder (2005a), (2006b) and the literature cited therein addressing the valuation of continuously sampled Asian options in the Black-Scholes model, these are new results, and they by no means exhaust the potential of the approach; the general picture is summarized in Section 10.

\section{Preliminaries on Lévy processes}

In this section we collect pertinent facts about the Lévy processes associated with generalized inverse Gaussian distributions.

\subsection{Lévy processes}

Lévy processes are processes of stationary, independent increments. Working on any filtered probability space $(\Omega, \mathcal{F}, \mathbb{F}, Q)$ whose filtration satisfies the usual conditions, we moreover assume the processes to be càdlàg (continuous from the right with left limits) so that they are progressively measurable with sample paths continuous almost everywhere. Recall that any Lévy process $X=\left(X_{t}, t \geq 0\right)$ is constructed from infinitely divisible distribution functions $f$; see Bauer (1996, Section 37) or Kallenberg (2002, Theorem 15.12). The construction is such 
that $f$ becomes the law of $X_{1}$, and up to a notion of equivalence this sets up a bijection between infinitely divisible distributions and Lévy processes.

\subsection{GIG Lévy processes}

A generalized inverse Gaussian $(G I G)$ Lévy process $X_{G I G(\xi)}$ is a Lévy process that depends on the parameter triple $\xi=(\gamma, \delta, \lambda)$ of reals. In the present paper we restrict the first two parameters to be positive: $\gamma, \delta>0$. Such a process is then constructed as indicated in Section 2.1 using the generalized inverse Gaussian distribution, $G I G(\xi)$, corresponding to $\xi$. The latter's Lebesgue density is given on the positive real line by

$$
d_{G I G(\xi)}(w)=\frac{1}{2} \frac{(\gamma / \delta)^{\lambda}}{K_{\lambda}(\gamma \delta)} w^{\lambda-1} \exp \left(-\frac{1}{2}\left(\frac{\delta^{2}}{w}+\gamma^{2} w\right)\right), \quad w>0 .
$$

Here $K_{a}$ is the $K$-Bessel function of any complex degree $a$; see Lebedev (1972, Chapter 5). From Lebedev (1972, Equation (5.10.25)), in particular recall the integral representation

$$
K_{a}(z)=\frac{1}{2}\left(\frac{z}{2}\right)^{a} \int_{0}^{\infty} w^{-a} \exp \left(-w-\frac{(z / 2)^{2}}{w}\right) \frac{\mathrm{d} w}{w},
$$

which holds for all complex $z$ with $\operatorname{Re}\left(z^{2}\right)>0$. These functions also enter into the Fourier transform of these densities by means of the weighted $K$-Bessel function $F_{\xi}$ defined by

$$
F_{\xi}(z)=\frac{K_{\lambda}(\delta \sqrt{\varphi(z)})}{(\delta \sqrt{\varphi(z)})^{\lambda}}
$$

for any complex $z$ with $\operatorname{Re}(z)<\gamma^{2} / 2$, where $\varphi \equiv \varphi_{\gamma}$ is given by $\varphi(z)=\gamma^{2}-2 z$. In fact, from Jørgensen (1982, Equation (2.9)) or Prause (1999, Lemma 1.38), we have

$$
M G(z)=\frac{1}{F_{\xi}(0)} F_{\xi}(z), \quad \operatorname{Re}(z)<\frac{1}{2} \gamma^{2},
$$

where $M G$ is the moment generating function of the time-1 value, $X_{G I G(\xi), 1}$, of $X_{G I G(\xi)}$,

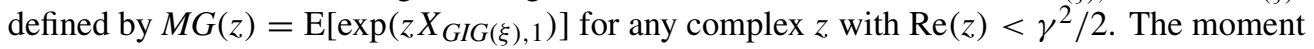
generating function, $M G_{t}$, of $X_{G I G(\xi)}$ at any time $t$ is obtained by raising $M G$ to its $t$ th power: $M G_{t}=M G^{t}$. This general property of Lévy processes (see Kallenberg (2002, Corollary 15.8)) enables a two-step computation of their moments. The first step is as follows.

Proposition 2.1. Let $X$ be any Lévy process whose first $N$ time-1 moments exist. Then its first $N$ time-t moments exist for any time $t$, and we have

$$
\mathrm{E}\left[X_{t}^{n}\right]=\sum_{k=1}^{n}(-1)^{k}(-t)_{k} \sum_{\ell=0}^{k-1} \frac{(-1)^{\ell}}{(k-\ell) ! \ell !} F_{k-\ell}^{n}(0)
$$

for any nonnegative integer $n \leq N$.

The concepts involved here are as follows. By $(a)_{k}$ we denote the $k$ th Pochhammer symbol of any complex $a$, which is recursively defined by $(a)_{0}=1$ and $(a)_{k+1}=(a+k)(a)_{k}$ for any nonnegative integer $k$. The $F_{m}^{n}$ are functions on the domain of definition of the time-1 moment generating function, $M G_{X}$, of $X$, and are recursively defined as follows: for any nonnegative 
integers $n, m \leq N$, let $F_{m}^{0}=M G_{X}^{m}$, the $m$ th power of $M G_{X}$, and let $F_{0}^{n}=M G_{X}^{(n)}$, the $n$th derivative of $M G_{X}$, and for such positive integers set

$$
F_{m}^{n}=\sum_{k=0}^{n}\left(\begin{array}{l}
n \\
k
\end{array}\right) F_{1}^{n-k} F_{m-1}^{k} .
$$

Hence, we have the multinomial expansion

$$
F_{m}^{n}=\sum_{L}\left(\begin{array}{l}
n \\
L
\end{array}\right) M G_{X}^{\left(\ell_{1}\right)} \cdots M G_{X}^{\left(\ell_{m}\right)}
$$

where the sum is over all $m$-tuples $L=\left(\ell_{1}, \ldots, \ell_{m}\right)$ of nonnegative integers with sum $|L|=$ $\ell_{1}+\cdots+\ell_{m}=n$; in particular, we obtain

$$
F_{m}^{n}(0)=\sum_{L}\left(\begin{array}{l}
n \\
L
\end{array}\right) \mathrm{E}\left[X_{1}^{\ell_{1}}\right] \cdots \mathrm{E}\left[X_{1}^{\ell_{m}}\right] .
$$

Proof of Proposition 2.1. The formula is established by differentiation of the $t$ th power of $M G_{X}$. This is seen to reduce to a verification of the differentiation formula

$$
\left(M G_{X}^{a}\right)^{(n)}=\sum_{k=1}^{n}(-1)^{k}(-a)_{k} M G_{X}^{a-k} U_{k}, \quad \text { where } U_{k}=\sum_{\ell=0}^{k-1} \frac{\left(-M G_{X}\right)^{\ell}}{(k-\ell) ! \ell !}\left(M G_{X}^{k-\ell}\right)^{(n)} .
$$

This result reduces the computation of the moments of GIG Lévy processes to that of their time-1 moments, and a direct integration of the pertinent densities as in Jørgensen (1982, Equation (2.16)) is then seen to yield the second step, as follows.

Proposition 2.2. All nonnegative moments of any GIG Lévy process $X_{G I G(\xi)}$ exist, and we have

$$
\mathrm{E}\left[X_{G I G(\xi), 1}^{n}\right]=\left(\frac{\delta}{\gamma}\right)^{n} \frac{K_{\lambda+n}(\gamma \delta)}{K_{\lambda}(\gamma \delta)}
$$

for any nonnegative integer $n$.

\section{Preliminaries on Laguerre reduction series}

In this section we assemble pertinent results on the Laguerre reduction series of stochastic functionals.

\subsection{Laguerre series}

In this section we recall pertinent properties of Laguerre polynomials from Lebedev (1972, Section 4) and Sansone (1991). The structural setting for them is a generalization of the classical Hilbert space of square-integrable functions. For any real $\alpha>-1$, let $L_{\alpha}^{2}(0, \infty)$ be the Hilbert space of all real-valued functions $F$ on the positive reals that are $\alpha$-square integrable, i.e. such that

$$
\|F\|_{\alpha}^{2}:=\int_{0}^{\infty} w_{\alpha}(x)|F|^{2}(x) \mathrm{d} x<\infty,
$$

with the weight $w_{\alpha}$ on $(0, \infty)$ given by $w_{\alpha}(x)=x^{\alpha} \exp (-x)$. Then $L_{\alpha}^{2}(0, \infty)$ carries the bilinear form $\langle,\rangle_{\alpha}$ given by

$$
\langle F, G\rangle_{\alpha}=\int_{0}^{\infty} w_{\alpha}(x) F(x) G(x) \mathrm{d} x,
$$


and an orthogonal basis for this Hilbert space is furnished by the $\alpha$-Laguerre polynomials, $L_{m}^{\alpha}(z)$. For any nonnegative integer $m$, these are given by

$$
L_{m}^{\alpha}(z)=\sum_{k=0}^{m} \alpha_{m, k} z^{k}, \quad \text { where } \alpha_{m, k}=\frac{(-1)^{k}}{k !}\left(\begin{array}{l}
m+\alpha \\
m-k
\end{array}\right),
$$

for any complex number $z$, and satisfy $\left\|L_{m}^{\alpha}\right\|_{\alpha}^{2}=\Gamma(m+\alpha+1) / m$ !. Expressing any $F$ in $L_{\alpha}^{2}(0, \infty)$ in this basis yields its $\alpha$-Laguerre series,

$$
F=\sum_{m=0}^{\infty} c_{m} L_{m}^{\alpha}, \quad \text { where } c_{m}=\frac{\left\langle F, L_{m}^{\alpha}\right\rangle_{\alpha}}{\left\langle L_{m}^{\alpha}, L_{m}^{\alpha}\right\rangle_{\alpha}}
$$

The $c_{m}$ are the $\alpha$-Laguerre coefficients of this series, whose convergence to $F$ is in $\alpha$-mean: $\lim _{M \rightarrow \infty}\left\|F-\sum_{m=0}^{M} c_{m} L_{m}^{\alpha}\right\|_{\alpha}=0$.

\subsection{Growth measures}

In this section we recall and modify the local growth measures $\delta$ and $\gamma$ of Schröder (2005a, Section 2.1) and Schröder (2006a, Section 2.2), which encode how Laguerre expandability is determined by local data. They apply to any complex-valued function $f$ on the positive reals and the idea of them is to describe the behaviour of $f$ at any point $a$ of the nonnegative real line or at $\infty$ by comparison with the behaviour of power maps or powers of the exponential function, respectively. The exponential growth order, $\delta_{a}(f)$, of $f$ near $a$ measures the rate of exponential decay of $f$ at $a$ and is defined as follows:

$$
\delta_{a}(f)=\sup \left\{\delta \in \mathbb{R}: \lim _{x \rightarrow a} \exp (\delta x) f(x)=0\right\} .
$$

The polynomial growth order, $\gamma_{a}(f)$, of $f$ near $a$ gives for meromorphic functions $f$ the degree of the leading terms of their Laurent series expansions in $a$. In general, it is defined as follows. If $a$ is finite then

$$
\gamma_{a}(f)=\sup \left\{\gamma \in \mathbb{R}: \lim _{x \rightarrow a} f(x) /(x-a)^{\gamma}=0\right\},
$$

and otherwise $\gamma_{\infty}(f)=\gamma_{0}\left(f^{*}\right)$, where $f^{*}(x)=f(1 / x)$. The respective sets of real numbers defining these growth orders may be empty, in which case the supremum of each is $-\infty$.

\subsection{Laguerre reduction series}

The notion of a Laguerre reduction series was developed in Schröder (2005a) as an alternative to Dufresne (2000), and Schröder (2006a) furnishes a common framework for both approaches. Adopting the setting of Section 3.1, the result which is pertinent and basic to the present paper is as follows.

Theorem 3.1. Let $Y$ be any positive random variable with probability density $g_{Y}$, and let the measurable function $\rho$ on the positive reals and the real $\alpha>-1$ be such that $\rho$ and $g_{Y} / w_{\alpha}$ are both in $L_{\alpha}^{2}(0, \infty)$. Then we have the absolutely convergent Laguerre reduction series representation

$$
\mathrm{E}[\rho(Y)]=\sum_{m=0}^{\infty} a_{m}\left\langle\rho, L_{m}^{\alpha}\right\rangle_{\alpha}
$$


whose error terms, $R_{M}=\sum_{m=M}^{\infty} a_{m}\left\langle\rho, L_{m}^{\alpha}\right\rangle_{\alpha}$, satisfy the inequality

$$
R_{M}^{2} \leq\|\rho\|_{\alpha}^{2} \sum_{m=M}^{\infty} a_{m}^{2}\left\|L_{m}^{\alpha}\right\|_{\alpha}^{2}
$$

The coefficients $a_{m}$ are the $\alpha$-Laguerre coefficients of $g_{Y} / w_{\alpha}$, namely

$$
a_{m}=\frac{\left\langle g_{Y} / w_{\alpha}, L_{m}^{\alpha}\right\rangle_{\alpha}}{\left\langle L_{m}^{\alpha}, L_{m}^{\alpha}\right\rangle_{\alpha}}=\sum_{k=0}^{m} \frac{\alpha_{m, k}}{\left\|L_{m}^{\alpha}\right\|_{\alpha}^{2}} \mathrm{E}\left[Y^{k}\right],
$$

given here in terms of the $\alpha_{m, k}$, the coefficients of $L_{m}^{\alpha}$, and the $\mathrm{E}\left[Y^{k}\right]$, the $k$ th moments of $Y$. Similarly,

$$
\left\langle\rho, L_{m}^{\alpha}\right\rangle_{\alpha}=\sum_{k=0}^{m} \alpha_{m, k} \int_{0}^{\infty} w_{\alpha}(y) \rho(y) y^{k} \mathrm{~d} y .
$$

The theorem is quoted directly from Schröder (2005a, Theorem 3.2 and Addendum 3.4) in the situation $\beta=\alpha$ and $\delta=1$ there, or Schröder (2006a, Theorem 5.3 and Proposition 5.1) in the situation where, moreover, $N=0$. It essentially asserts the validity of taking the expectation through the series for $\rho g_{Y}$ obtained using the expansion of $g_{Y} / w_{\alpha}$ in terms of $\alpha$-Laguerre polynomials. Its proof reduces to establishing the error estimates, and these follow on combining a Cauchy-Schwarz estimate for the remainder term functionals, $R_{M}$, using the Parseval identity in $L_{\alpha}^{2}(0, \infty)$ for the Laguerre expansion of $g_{Y} / w_{\alpha}$. A complete argument was given in Schröder (2006a, Section 5.5).

\subsection{Expandability criterion}

Using the concepts and notation of Section 3.3, in this section we develop a new sufficient criterion for the Theorem 3.1 Laguerre reduction series to exist. In addition to the local growth measures of Section 3.2, it is given in terms of the moment generating function, $M G_{Y}$, of the positive random variable $Y$. This function is defined by $M G_{Y}(z)=\mathrm{E}[\exp (z Y)]$ for any complex $z$ with $\operatorname{Re}(z)<\sigma_{\mathrm{sc}}(Y)$, where

$$
\sigma_{\mathrm{sc}}(Y)=\sup \{\operatorname{Re}(z): \mathrm{E}[\exp (z Y)] \text { is finite }\}
$$

denotes the abscissa of convergence of $M G_{Y}$. With these concepts, our results are as follows.

Theorem 3.2. In the setting of Theorem 3.1, a sufficient condition for $g_{Y} / w_{\alpha}$ to be in $L_{\alpha}^{2}(0, \infty)$ is that the following two inequalities be satisfied:

$$
\sigma_{\mathrm{sc}}(Y)>\frac{1}{2}, \quad \alpha+1<2\left(\gamma_{\infty}\left(\left|M G_{Y}^{-}\right|\right)-1\right),
$$

where $M G_{Y}^{-}(z)=M G_{Y}(-z)$ for any complex $z$ with $\operatorname{Re}(z)>-\sigma_{\mathrm{sc}}(Y)$.

Addendum 3.1. We have $\gamma_{\infty}\left(\left|M G_{Y}^{-}\right|\right)=\infty$ if $\left|M G_{Y}^{-}\right|(z)$ decays to 0 exponentially as $|z|$ tends to $\infty$.

We precede the proof with two results of independent interest. They explicitly relate the growth measures $\delta_{\infty}$ and $\gamma_{0}$ of $g_{Y}$, the density of $Y$, to the moment generating function of $Y$.

Lemma 3.1. We have $\delta_{\infty}\left(g_{Y}\right)=\sigma_{\mathrm{sc}}(Y)$.

Lemma 3.2. We have $\gamma_{0}\left(g_{Y}\right)=\gamma_{\infty}\left(\left|M G_{Y}^{-}\right|\right)-1$. 
Let us prove these two results first. Lemma 3.1 is immediate from the definitions, whereas Lemma 3.2 is a translation of the Abel asymptotics for $M G_{Y}$ at $\infty$. In fact, Doetsch (1971, Satz 3, pp. 503f.) showed that if $\mu>1$ is any real such that

$$
M G_{Y}(z) \sim \frac{A}{z^{\mu}} \quad \text { as }|z| \rightarrow \infty,
$$

for a nonzero constant $A$, then there exists a nonzero constant $B$ such that

$$
g_{Y}(y) \sim B y^{\mu-1} \quad \text { as } y \downarrow 0 .
$$

With the maximal such $\mu$ equal to the polynomial growth order $\gamma_{\infty}\left(\left|M G_{Y}^{-}\right|\right)$, the proof of Lemma 3.2 is complete.

Proof of Theorem 3.2. Sufficient for $g_{Y} / w_{\alpha}$ to be in $L_{\alpha}^{2}(0, \infty)$ is that, firstly, $g_{Y}^{2} / w_{\alpha}$ is integrable on any compact subset of the positive reals and, secondly, that the following two inequalities are satisfied: $2 \delta_{\infty}\left(g_{Y}\right)>1$ and $\alpha+1<2 \gamma_{0}\left(g_{Y}\right)$. Using the above two lemmas, these two inequalities translate into those of the theorem. Sufficient for the integrability of $g_{Y}^{2} / w_{\alpha}$ on compact sets, on the other hand, is the integrability of $g_{Y}$ there. This is implied by the finiteness of $M G_{Y}$ at any $z$ with $\operatorname{Re}(z)<\sigma_{\mathrm{sc}}(Y)$; thus, the proof of Theorem 3.2 is complete.

\section{Basic setting}

The aim guiding the development of this paper is to study discretely sampled stochastic functionals of Lévy processes in order to understand the continuously sampled ones, and vice versa. To proceed we let $X$ be any Lévy process as described in Section 2.1 and consider it on the subset $\left[t_{0}, T\right]$ of the nonnegative real line. To speak of discretely sampled functionals then requires us to choose finitely many (in fact $N$ ) points $t_{m}$ with $t_{1}<t_{2}<\cdots<t_{N}$ in this time interval, where we let $N \geq 1$ and assume that $t_{N}=T$. It also means considering expectations of the form

$$
\mathrm{E}\left[R\left(X_{t_{1}}, \ldots, X_{t_{N}}\right) \mid \mathcal{F}_{t}\right],
$$

which are conditional on $\mathcal{F}_{t}$, the information available at any time $t$ in $\left[t_{0}, T\right]$. Here $R$ is any $\mathcal{F}_{T}$-measurable, complex-valued map on the $N$-fold product of the codomain of $X$. We will return to this general situation in Section 10 . Here, we let $X$ be any positive process and specialize the maps $R$ as follows. We concentrate on maps that factorize over lower-dimensional ones; in fact, we consider maps that factorize over one-dimensional ones as follows:

$$
R=\rho \circ \sigma_{N}
$$

Here $\rho$ is a map on the positive reals and $\sigma_{N}$ is any real-valued map on $\mathbb{R}^{N}$ sending the positive cone there into the positive reals: $\sigma_{N}\left(\left(\mathbb{R}_{>0}\right)^{N}\right) \subseteq \mathbb{R}_{>0}$. Specializing further, we finally take $\sigma_{N}$ to be any element of the dual space of $\mathbb{R}^{N}$. It then corresponds to an $N$-tuple $a=\left(a_{1}, \ldots, a_{N}\right)$ of positive reals, and we let

$$
\Sigma_{N}^{a}:=\sigma_{N}\left(X_{t_{1}}, \ldots, X_{t_{N}}\right)=\sum_{m=1}^{N} a_{m} X_{t_{m}} .
$$

While not restricted to this context, our motivation for considering functionals of the final form comes from the risk-neutral valuation of contingent claims. To conclude this section, as well as to fix ideas, we now present four examples of Asian-type contingent claims. 
Example 4.1. Consider options on averages of prices such as the discretely monitored Asian options. In the above setting, these are contingent claims with time- $T$ payoff $\rho\left(\Sigma_{N}^{a}\right)$, where $\rho$ is the payoff of any put or call option, the weights $a_{m}$ are all equal to $1 / N$, and $X$ is interpreted as the price process in continuous time of any asset under any equivalent martingale measure. Assuming a deterministic short rate, the above stochastic functionals give the risk-neutral values of these options on discounting. This example is considered in more detail in Section 9.

Example 4.2. Consider interest rate derivatives. Here the above discussion in particular pertains to the discrete-time construction of new rates, $\Sigma_{N}^{a}$, from rates $X$ modelled in continuous time, and to the risk-neutral valuation of contingent claims written on them. We give a more detailed discussion of these futures-type contingent claims in Section 7. Our approach can also be adapted to the interest rate derivatives presented in Musiela and Rutkowski (1997, Section 10). Assuming Lévy process term structures, as it stands this approach extends to the risk-neutral valuation of swap-type or swaption-type contingent claims. Higher-dimensional versions of it will be needed in valuing caps or captions; these are sketched in Section 10.

Example 4.3. Consider embedded options in insurance contracts and, in particular, indexlinked life insurance contracts. To place these in the above setting, we note that a simple version of the second type of contract asks for $N$ premium payments to take place at the points in time $t_{m}$. Any $m$ th premium is split: an amount $a_{m}$ is immediately invested in a mutual fund and the remainder furnishes the premium payment for a standard life insurance contract. If the contract terminates at time $t$ then its payoff consists of two components: the stipulated amount from its life insurance component and the excess over this amount of the time- $t$ value of the mutual fund portfolio built up until time $t$. Computation of the fair prices of the contracts requires the risk-neutral values of these embedded options; see Aase Nielsen and Sandmann (1995), (1996), (2002) for the precise statements and some calculations. To cast this last problem in our framework reduces to constructing the value of the mutual fund portfolio as a random variable, $\Sigma_{N}^{a}$, for any fixed time $t$. This value is explicitly given by $\sum_{t_{m} \leq t} a_{m}\left(S_{t} / S_{t_{m}}\right)$, where $S_{u}$ denotes the time- $u$ value of the mutual fund. To complete the picture, let $X$ be any process whose time- $t_{m}$ value coincides with the respective time- $t_{m}$ mutual fund price quotient $S_{t} / S_{t_{m}}$.

Example 4.4. Consider contingent claim valuation in stochastic volatility models of HullWhite type. By this we understand models with the following two properties. Firstly, $X$ is interpreted as generalizing the variance of an asset, $\Sigma_{N}^{a}$ thus generalizing the discretely sampled average variance. Secondly, the valuation of any contingent claim reduces to computing the above expectations with $\rho(v)$ given as the risk-neutral, time- $t$ value of the contingent claim under consideration, conditional on having $\Sigma_{N}^{a}=v$ for any real $v>0$. While the construction of models with these two properties is straightforward in principle, the condition of $X$ being a Lévy process is too strict. For instance, it does not apply to the Ornstein-Uhlenbeck process used to model variance in Hull and White (1987) or the generalized Ornstein-Uhlenbeck processes used to do so in Barndorff-Nielsen and Shephard (2001). However, these processes are stochastic integrals with respect to Lévy process integrators, and this makes it possible to apply to them the theory developed for Lévy processes in the present paper, with the necessary changes; see Schröder (2005c).

\section{Convergence theorems}

In this section we address the first main step of our approach. Working in the setting of Section 4, we study the convergence of discretely sampled stochastic functionals of positive 
Lévy processes to the continuously sampled ones as sample size increases. The main results, Theorems 5.2 and 5.3, give explicit estimates for the rate of this convergence, and thus in principle reduce the study of the continuously sampled functionals to that of the discretely sampled ones.

\subsection{Basic setting}

Adopting the principal setting of Section 4 , we focus on equipartitions of $\left[t_{0}, T\right]$ by the points $t_{m}=t_{0}+m \tau_{N}$, where $\tau_{N}=\left(T-t_{0}\right) / N$, and associate with the positive Lévy process $X$ the random variables $\Sigma_{N}(X)$ and $A_{t, T}(X)$, for any nonnegative reals $t<T$, according to

$$
\Sigma_{N}(X)=\frac{1}{N} \sum_{m=1}^{N} X_{t_{m}} \quad \text { and } \quad A_{t, T}(X)=\frac{1}{T-t} \int_{t}^{T} X_{u} \mathrm{~d} u .
$$

The latter are well defined since, by hypothesis, $X$ has sample paths which are continuous almost everywhere. We call the functionals of type $\Sigma_{N}(X)$ discretely sampled and those of type $A_{t, T}(X)$ continuously sampled. We will work with those that are explicitly given by the expectations

$$
\mathrm{E}\left[\rho\left(\Sigma_{N}(X)\right) \mid \mathcal{F}_{t}\right] \quad \text { and } \quad \mathrm{E}\left[\rho\left(A_{t_{0}, T}(X)\right) \mid \mathcal{F}_{t}\right]
$$

which are conditional on time- $t$ information $\mathcal{F}_{t}$ for any $t$ in $\left[t_{0}, T\right)$, and moreover assume the maps $\rho$ on the positive reals to be such that these expectations are well defined and finite. Since we have convergence of $\Sigma_{N}(X)$ to $A_{t_{0}, T}(X)$ as $N$ tends to $\infty$, we should, under continuity assumptions on $\rho$, expect convergence of the discretely sampled stochastic functionals to the continuously sampled ones with increasing sample size, i.e. $\lim _{N \rightarrow \infty} \Delta_{t, N}(X)=0$ with

$$
\Delta_{t, N}(X):=\mathrm{E}\left[\rho\left(\Sigma_{N}(X)\right)-\rho\left(A_{t_{0}, T}(X)\right) \mid \mathcal{F}_{t}\right] .
$$

Our principal finding is that we can in fact make explicit the rate of this convergence if we place on $\rho$ additional differentiability assumptions such as the following (referred to as condition (R)).

(R) The map $\rho$ on the positive reals is almost everywhere differentiable and its derivative is bounded by a constant $M_{\rho}$.

\subsection{Main convergence results}

Using the notation and concepts of Section 5.1, in this section we formulate and discuss our main results concerning the convergence of $\Delta_{t, N}$, in three steps. As a first step, we linearize the problem as follows.

Theorem 5.1. In the setting of Section 5.1, assuming the validity of condition $(R)$ in particular, there exists a random variable $R$ which is bounded almost everywhere by $M_{\rho}$ and is such that

$$
\Delta_{t, N}(X)=\mathrm{E}\left[\left(\Sigma_{N}(X)-A_{t_{0}, T}(X)\right) R \mid \mathcal{F}_{t}\right] .
$$

This is proved by constructing $R$ from, essentially, the first-order Taylor series expansion of $\rho$, as

$$
R=R_{1}(A, \Sigma), \quad \text { where } R_{1}(a, \sigma)=\int_{0}^{1} \rho^{\prime}((1-w) a+w \sigma) \mathrm{d} w
$$

for any reals $a$ and $\sigma$ and we have simplified the notation using $\Sigma \equiv \Sigma_{N}(X)$ and $A \equiv A_{t_{0}, T}(X)$. In fact, if $A$ and $\Sigma$ were constant then this would just be the error term of the first-order Taylor expansion of the value of $\rho$ at $\Sigma$ around the point $A$. In the general case, however, we proceed 
as follows. Condition the expectation defining $\Delta \equiv \Delta_{t, N}(X)$ on $A$ and write it as a double integral. At this point handle the $\rho$-difference factor of the integrand as above using first-order Taylor expansions. Then reverse the conditioning steps to obtain the theorem's representation of $\Delta$ with the above random variable $R$. Strictly speaking, this construction determines $R$ only outside the inverse image under $A$ of the points of nondifferentiability of $\rho$. This, however, is a null set. The proof of Theorem 5.1 is thus complete.

As a first consequence, this result enables a decomposition, $\Delta_{t, N}(X)=\Delta_{\mathrm{ns}}+\Delta_{\mathrm{s}}$, of $\Delta$ into a part, $\Delta_{\mathrm{ns}}$, of a nonstochastic nature and a part, $\Delta_{\mathrm{S}}$, of a stochastic nature:

$$
\begin{aligned}
\Delta_{\mathrm{ns}} & =\frac{1}{T-t_{0}} \mathrm{E}\left[\left(\tau_{N} \sum_{t_{m} \leq t} X_{t_{m}}-\int_{t_{0}}^{t} X_{u} \mathrm{~d} u\right) R \mid \mathcal{F}_{t}\right], \\
\Delta_{\mathrm{s}} & =\frac{1}{T-t_{0}} \mathrm{E}\left[\left(\tau_{N} \sum_{t_{m}>t} X_{t_{m}}-\int_{t}^{T} X_{u} \mathrm{~d} u\right) R \mid \mathcal{F}_{t}\right] .
\end{aligned}
$$

Majorizing $\Delta_{t, n}(X)$ thus reduces to majorizing $\Delta_{\mathrm{ns}}$ and $\Delta_{\mathrm{s}}$ separately, and in doing so we moreover assume that $t=t_{n_{t}}$ for some nonnegative integer $n_{t} \leq N$, for simplicity.

Majorizing $\Delta_{\mathrm{ns}}$ reduces to a standard piece of numerical integration. Proceeding along the lines of, for example, Hämmerlin and Hoffmann (1989, Chapter 7, Section 1), our result is as follows.

Theorem 5.2. In the above setting, assuming the validity of condition $(R)$ in particular, we have

$$
\left|\Delta_{\mathrm{ns}}\right| \leq M_{\rho} \frac{t-t_{0}}{T-t_{0}} \omega_{h}\left(\tau_{N}\right)
$$

Here $\omega_{h}$ is the modulus of continuity of the function on $\left[t_{0}, t\right]$ given by $h(u)=X_{u}$, i.e. $\omega_{h}(\delta)=$ $\sup \left\{|h(x)-h(y)|: x, y \in\left[t_{0}, t\right],|x-y| \leq \delta\right\}$ for any real $\delta>0$.

This result enables us to develop a first intuition about the size of $\Delta$. In fact, if $h$ were differentiable on $\left[t_{0}, t\right]$ then we would clearly have $\omega_{h}(\delta) \leq(\delta / 2)\left\|h^{\prime}\right\|_{\infty}\left(t-t_{0}\right)$, where $\left\|h^{\prime}\right\|_{\infty}=$ $\sup \left\{\left|h^{\prime}(u)\right|: u \in\left[t_{0}, t\right]\right\}$. This suggests that at least the summand $\Delta_{\mathrm{ns}}$ of $\Delta_{t, N}(X)$ behaves like a scalar multiple of $1 / N$ as $N$ tends to $\infty$.

The majorization of $\Delta_{S}$, on the other hand, is based on stochastic information. Our result here, whose proof is presented in Section 5.4, is the following moment estimate.

Theorem 5.3. In the above setting, assuming the validity of condition $(R)$ in particular, we have

$$
\left|\Delta_{\mathrm{s}}\right| \leq M_{\rho} \mathrm{E}\left[X_{1}\right]\left(T-t_{0}\right) \frac{N-n_{t}}{2 N^{2}} .
$$

A number of ways of extending our results to functionals of random variables of a more general nature are also conceivable. They immediately extend, for example, to stochastic functionals of a finite number of random variables $\Sigma_{N}(X)$ in an obvious way.

\subsection{GIG Lévy process case}

This section is the specialization of Section 5.2 to the case in which $X$ is a GIG Lévy process $X_{G I G(\xi)}$ with parameters $\xi=(\lambda, \gamma, \delta)$, as described in Section 2.2. We moreover let $t=t_{0}$, and thus focus on $\Delta_{N}(X)$, the approximation error with no nonstochastic parts, given by

$$
\Delta_{N}(X)=\mathrm{E}\left[\rho\left(\Sigma_{N}(X)\right)-\rho\left(A_{t_{0}, T}(X)\right) \mid \mathcal{F}_{t_{0}}\right] .
$$


According to Theorem 5.3, the moment estimate of the approximation error then takes the following explicit form.

Theorem 5.4. In the setting of Sections 5.1 and 5.2, assuming the validity of condition $(R)$ in particular, for any positive integer $N$ we have

$$
\left|\Delta_{N}\left(X_{G I G(\xi)}\right)\right| \leq \frac{c(\xi)}{2 N},
$$

where

$$
c(\xi)=\left(T-t_{0}\right) M_{\rho} \frac{\delta}{\gamma} \frac{K_{\lambda+1}(\gamma \delta)}{K_{\lambda}(\gamma \delta)}
$$

and $K_{v}$ denotes the $K$-Bessel function of order $v$.

Theorem 5.4 follows on substitution into Theorem 5.3 of the explicit formula for the first time-1 moment of $X_{G I G(\xi)}$ from Proposition 2.2.

To interpret this result, the estimate predicts an initial accuracy which is solely determined by the constant $c(\xi)$. This initial accuracy is then predicted to improve by order $1 / N$, the reciprocal of the number of sample points, $N$.

Examples seem to indicate that, to within an accuracy of a few decimal places, the convergence of discretely sampled contingent claim values to continuously sampled ones can be achieved using a relatively small number of sample points. Their number seems to increase exponentially in the required order of accuracy.

\subsection{Proof of the moment estimate}

This section establishes the estimate of Theorem 5.3 for $\Delta_{\mathrm{S}}$. In particular, recalling the random variable $R$ constructed in Theorem 5.1, this is based on the following result.

Lemma 5.1. We have

$$
\Delta_{\mathrm{s}}=\frac{N-n_{t}}{N} \mathrm{E}\left[R A_{0, \tau_{N}}(X) \mid \mathcal{F}_{t}\right]
$$

where $A_{0, \tau_{N}}(X)$ is independent of the time-t information $\mathcal{F}_{t}$.

Proof. Using the simplified notation $\tau \equiv \tau_{N}$, in the definition of $\Delta_{\mathrm{S}}$ we express the integral over $[t, T]$ as a sum of integrals over the intervals $\left[t_{\ell-1}, t_{\ell}\right]$ of length $\tau$ and, looking at time increments in any one of them, make the change of variable $w(u)=u-t_{\ell-1}$. We thus obtain

$$
\Delta_{\mathrm{s}}=\frac{1}{T-t_{0}} \sum_{\ell=n_{t}+1}^{N} E_{\ell}
$$

where the $E_{\ell}$ are the expectations

$$
E_{\ell}=\mathrm{E}\left[\left(\tau\left(X_{t_{\ell}}-X_{t_{\ell-1}}\right)-\int_{0}^{\tau}\left(X_{w+t_{\ell-1}}-X_{t_{\ell-1}}\right) \mathrm{d} w\right) R \mid \mathcal{F}_{t}\right] .
$$

We first analyse any one difference $X_{t_{\ell}}-X_{t_{\ell-1}}=\left(X_{t_{\ell}}-X_{t}\right)-\left(X_{t}-X_{t_{\ell-1}}\right)$ by successively restarting $X$ in two steps, as follows. On restarting at time $t$, as a first step, the difference is equal in law to $X_{t_{\ell}-t}-X_{t_{\ell-1}-t}$ and is independent of $\mathcal{F}_{t}$. On restarting this restarted process at time $t_{\ell-1}-t$, as a second step, the difference is equal in law to $X_{t_{\ell}-t_{\ell-1}}=X_{\tau}$. Working at 
the process level, analogous restarting arguments apply to the integrated process part, and we hence have

$$
E_{\ell}=\mathrm{E}\left[\left(\tau X_{\tau}-\int_{0}^{\tau} X_{u} \mathrm{~d} u\right) R \mid \mathcal{F}_{t}\right]=: E^{*},
$$

independently of $\ell$, with the random variables in round brackets independent of the time- $t$ information $\mathcal{F}_{t}$. Rewriting the differences as integrals and reversing time then gives

$$
E^{*}=\mathrm{E}\left[R \int_{0}^{\tau}\left(X_{\tau}-X_{\tau-u}\right) \mathrm{d} u \mid \mathcal{F}_{t}\right] .
$$

Using the Markov property of $X$, the processes $\left(X_{\tau}-X_{\tau-u}\right)_{u \in[0, \tau]}$ and $\left(X_{w}\right)_{w \in[0, \tau]}$ are equal in law. Noting that there are $N-n_{t}$ summands $E^{*}$ in $\Delta_{s}$ completes the proof of Lemma 5.1.

Proof of Theorem 5.3. Using the simplified notation $\tau \equiv \tau_{N}$, by inspection we are reduced to showing that

$$
\left|\Delta_{\mathrm{S}}\right| \leq M_{\rho} \frac{N-n_{t}}{N} \mathrm{E}\left[A_{0, \tau}(X)\right]
$$

where

$$
\mathrm{E}\left[A_{0, \tau}(X)\right]=\frac{1}{2} \tau \mathrm{E}\left[X_{1}\right] .
$$

Given the boundedness in absolute value of $R$ by $M_{\rho}$, the first inequality is immediate from Lemma 5.1, and it thus remains to establish the expression for the first moment of $A_{0, \tau}(X)$. To do so we first apply Tonelli's theorem, to obtain

$$
\mathrm{E}\left[A_{0, \tau}(X)\right]=\frac{1}{\tau} \int_{0}^{\tau} \mathrm{E}\left[X_{u}\right] \mathrm{d} u .
$$

With $M G$ denoting the time-1 moment generating function of $X$, we have

$$
\mathrm{E}\left[X_{u}\right]=\frac{\mathrm{d}}{\mathrm{d} u} M G^{u}(0)=u M G^{u-1}(0) M G^{\prime}(0)
$$

Hence, integration yields the result and the proof of Theorem 5.3 is complete.

\section{Normalization of stochastic functionals}

As the second main step of our approach to stochastic functionals, in this section we develop a normalization procedure. Thus, taking up the discussion of Section 4, as regards working with the Section 4 functionals

$$
\mathrm{E}\left[R\left(X_{t_{1}}, \ldots, X_{t_{N}}\right) \mid \mathcal{F}_{t}\right],
$$

we would like the random variables $X_{t_{m}}$ to be independent. Unfortunately, in most cases this is not only false but also too strong a property to require from the point of view of applications. Independence of their time increments, on the other hand, is a property which not only often holds for the $X_{t_{m}}$ but is often true in applications as well. This suggests regarding the above functionals of the $X_{t_{m}}$ as functionals of the time increments of these variables. While we return to the general situation in Section 10, we exemplify this perspective here by concentrating on the class of functionals arrived at in Section 4 . With $X$ now any Lévy process, these are given by the expectations

$$
\mathrm{E}\left[\rho\left(\Sigma_{N}^{a}\right) \mid \mathcal{F}_{t}\right], \quad \text { where } \Sigma_{N}^{a}=\sum_{m=1}^{N} a_{m} X_{t_{m}},
$$


and are conditional on the time- $t$ information $\mathcal{F}_{t}$ for any $t$ in $\left[t_{0}, T\right]$. Here $\rho$ is any measurable function now on (all) the reals and $a=\left(a_{1}, \ldots, a_{N}\right)$ is now any $N$-tuple of reals. The principal idea is to develop a normalizing procedure for these functionals which represents them in terms of independent random variables. In the present context, our principal result is as follows.

Proposition 6.1. In the above setting there is a method of construction that depends on time $t$ and yields maps $\rho_{t}$ on the reals, random variables $Y_{1}, \ldots, Y_{n_{t}}$, reals $\gamma_{1}, \ldots, \gamma_{n_{t}}$, and positive reals $\tau_{1}, \ldots, \tau_{n_{t}}$ such that the following two assertions hold.

1. $\mathrm{E}\left[\rho\left(\Sigma_{N}^{a}\right) \mid \mathcal{F}_{t}\right]=\mathrm{E}\left[\rho_{t}\left(\Xi_{n_{t}}^{\gamma}\right)\right]$, where $\Xi_{n_{t}}^{\gamma}=\sum_{k=1}^{n_{t}} \gamma_{k} Y_{k}$.

2. The $Y_{k}$ are independent of each other and the time-t information $\mathcal{F}_{t}$, and satisfy $Y_{k} \stackrel{\mathrm{D}}{=} X_{\tau_{k}}$, where ' $\stackrel{\mathrm{D}}{=}$ 'denotes equality in law.

Addendum 6.1. If the coefficients $a_{m}$ are positive, then the $\gamma_{k}$ are positive. If, moreover, the $X_{t_{m}}$ are positive, then the random variables $\Xi_{n_{t}}^{\gamma}$ are positive.

We begin the construction described in Proposition 6.1 by decomposing $\Sigma_{N}^{a}$ into a part deterministic at time $t$ and a part stochastic at time $t$. We do this, however, in a normalizing way. By subtracting suitable multiples of $X_{t}$, we represent the latter part as

$$
\Xi=\sum_{t_{m}>t} a_{m}\left(X_{t_{m}}-X_{t}\right),
$$

i.e. as a sum of $n_{t}$ terms, meaning that the former part becomes $A_{t}=\sum_{m=1}^{N} a_{m} X_{t_{m} \wedge t}$. Defining the function $\rho_{t}$ on the reals by

$$
\rho_{t}(x)=\rho\left(A_{t}+x\right), \quad x \in \mathbb{R},
$$

we have $\rho\left(\Sigma_{N}^{a}\right)=\rho_{t}(\Xi)$, and the task is to construct the random variable $\Xi$ from random variables $Y_{k}$ as above.

This we achieve by consecutively restarting $X$ and using its Markov property. First restart $X$ at time $t$, to obtain the process $X^{*}$, and thus express $\Xi$ in law by then summing the values $X^{*}\left(\tau_{m}^{*}\right)$ with $\tau_{m}^{*}=t_{m}-t$. As a next step, represent each $X^{*}\left(\tau_{m}^{*}\right)$ as a telescoping sum of the consecutive differences $Y_{k}$ given by

$$
Y_{k}=X_{\tau_{m_{t}+k}^{*}}^{*}-X_{\tau_{m_{t}+k-1}^{*}}^{*}, \quad 1 \leq k \leq n_{t},
$$

where $m_{t}=N-n_{t}$ is the largest index $m$ such that $t_{m} \leq t$, and where we formally set $\tau_{0}^{*}=0$. On interchanging the order of summation, we obtain

$$
\Xi \stackrel{\mathrm{D}}{=} \sum_{\ell=1}^{n_{t}} a_{m_{t}+\ell} \sum_{k=1}^{\ell} Y_{k}=\sum_{k=1}^{n_{t}} \gamma_{k} Y_{k}
$$

where we have set

$$
\gamma_{k}=\sum_{\ell=k}^{n_{t}} a_{m_{t}+\ell} .
$$

Here the $Y_{k}$ are independent as increments of the Lévy process $X^{*}$, and they are independent of the time-t information $\mathcal{F}_{t}$ since $X^{*}$ has this property. Keeping track of the explicit differences defining them, the $Y_{k}$ moreover satisfy

$$
Y_{k} \stackrel{\mathrm{D}}{=} X_{\tau_{k}}, \quad \text { where } \tau_{k}=\tau_{m_{t}+k}^{*}-\tau_{m_{t}+k-1}^{*},
$$


whence $\tau_{1}=t_{m_{t}+1}-t$ and $\tau_{k}=t_{m_{t}+k}-t_{m_{t}+k-1}$ for $k \geq 2$. This completes the proof of Proposition 6.1, and the assertions of Addendum 6.1 are obvious by inspection.

Writing $n \equiv n_{t}$ and defining

$$
\Xi_{m, n}^{\gamma}=\sum_{k=m}^{n} \gamma_{k} Y_{k}
$$

for any positive integer $m \leq n$, we record three consequences of the Proposition 6.1 normalization procedure. The first is immediate from the above construction of the $Y_{k}$.

Proposition 6.2. The above random variables $Y_{m}$ and $\Xi_{m+1, n}^{\gamma}$ are independent, $\Xi_{m, n}^{\gamma}$ is independent of time- $\tau_{m-1}$ information, and we have $\Xi_{m, n}^{\gamma}=\gamma_{m} Y_{m}+\Xi_{m+1, n}^{\gamma}$.

The independence of the $Y_{k}$ translates into a product structure for the moment generating functions, as follows.

Proposition 6.3. The moment generating function, $M G_{\Xi}$, of any $\Xi \equiv \Xi_{m, n}^{\gamma}$ is expressible as the product,

$$
M G_{\Xi}=\prod_{k=m}^{n} M G_{\gamma_{k} Y_{k}}=\prod_{k=m}^{n} \gamma_{k}^{*}\left(M G_{Y_{k}}\right),
$$

of the moment generating functions of the summands of $\Xi$, and its domain of definition is the intersection of the domains of definition of the latter $n-m$ functions.

Here we have let $\gamma_{k}^{*}\left(M G_{Y_{k}}\right)(z)=M G_{Y_{k}}\left(\gamma_{k} z\right)$, meaning that $\gamma_{k}^{*}\left(M G_{Y_{k}}\right)(z)=M G_{\gamma_{k} Y_{k}}(z)$, the moment generating function of any $\gamma_{k} Y_{k}$.

On differentiation of this product representation, we finally obtain the following characterization of the moments of $\Xi$ in terms of those of $X$ itself.

Proposition 6.4. Let $M$ be any nonnegative integer. For the Mth moment of any $\Xi_{m, n}^{\gamma}$ we then have the recurrence rule

$$
\mathrm{E}\left[\left(\Xi_{m, n}^{\gamma}\right)^{M}\right]=\sum_{k=0}^{M}\left(\begin{array}{c}
M \\
k
\end{array}\right) \gamma_{m}^{k} \mathrm{E}\left[X_{\tau_{m}}^{k}\right] \mathrm{E}\left[\left(\Xi_{m+1, n}^{\gamma}\right)^{M-k}\right],
$$

if $m<n$, and the multinomial expansion

$$
\mathrm{E}\left[\left(\Xi_{m, n}^{\gamma}\right)^{M}\right]=\sum_{|J|=M}\left(\begin{array}{c}
M \\
J
\end{array}\right) \gamma^{J} \prod_{k=m}^{n} \mathrm{E}\left[X_{\tau_{k}}^{j_{k}}\right]
$$

where the sum is over all $(n-m)$-tuples $J=\left(j_{m}, \ldots, j_{n}\right)$ of nonnegative integers with $|J|=j_{m}+\cdots+j_{n}=M$, and $\gamma^{J}=\prod_{k=m}^{n} \gamma_{k}^{j_{k}}$.

\section{Applications to futures-type stochastic functionals}

In this section we focus on a class of discretely sampled stochastic functionals originating in interest rate futures contracts. Illustrating as well as extending the results of Section 6 , we develop, again in three stages, both normalizations for such stochastic functionals and explicit formulae for their values.

Let $\left[t_{0}, T\right]$ once more be any compact subinterval of the nonnegative reals, and for any integer $N \geq 1$ choose in it $N$ time points $t_{k}$ with $t_{1} \leq t_{2} \leq \cdots \leq t_{N}=T$. Let $Z$ be any process 
on $\left[t_{0}, T\right]$, and for any time $t$ in this interval consider the functionals given by the expectations conditional on time- $t$ information $\mathcal{F}_{t}$ as follows:

$$
\mathrm{E}\left[\rho\left(R_{T}\right) \mid \mathcal{F}_{t}\right] .
$$

Here $\rho$ is any measurable function on the reals and

$$
R_{T}=R_{0} \prod_{t_{k} \leq T}\left(1+a_{k} Z_{t_{k}}\right)
$$

for any reals $R_{0}$ and $a_{k}$. Then $R_{t}$ is the part of $R_{T}$ which is deterministic at time $t$, while the remaining $n_{t}$ factors are stochastic. Expanding the product of stochastic factors yields

$$
R_{T}=R_{t}+R_{t} \sum_{k=1}^{n_{t}} \sum_{J_{k}} a_{J_{k}} \pi_{J_{k}}(Z),
$$

where

$$
\pi_{J_{k}}(Z)=\prod_{\ell=1}^{k} Z_{t_{j_{\ell}}} .
$$

In this expression for $R_{T}$, any $k$ th summand of its first sum represents the $k$ th symmetric polynomial in $a_{\ell} Z_{t_{\ell}}$ with indices $\ell$ such that $t_{\ell}>t$. Denoting by $\ell_{t}=\max \left\{\ell: t_{\ell} \leq t\right\}$ the index of the last time, $t_{\ell}$, before or equal to $t$, the second sum is over all $k$-tuples $J_{k}=\left(j_{1}, \ldots, j_{k}\right)$ of integers with $\ell_{t}+1 \leq j_{1}<j_{2}<\cdots<j_{k} \leq N$, and for any of these $k$-tuples we let $a_{J_{K}}=\prod_{\ell=1}^{k} a_{j_{\ell}}$.

The definition of these functionals is motivated by the valuation of futures, whose time- $t$ values are equal to the risk-neutral expectations of their delivery values conditional on the time- $t$ information $\mathcal{F}_{t}$; see Duffie (1996, Section 8C). This corresponds to the case in which $\rho$ is the identity map, and, by thinking of $R_{T}$ as a new synthetic interest rate to be delivered at time $T$, we can in fact interpret its representations above as follows: they show how the rate delivered at time $T$ is constructed from the finitely many standard interest rates $Z_{t_{k}}$. Examples of such constructions include the futures contracts indexed on the Euro overnight index average; see LIFFE (2004).

A principal problem in working with these representations of $R_{T}$, however, is that the $Z_{t_{k}}$ are in general not independent. To adapt the technique developed for this purpose in Section 6 , we therefore specialize to the two cases in which $Z$ itself is respectively a Lévy process and an exponential Lévy process. Here the idea is to express any $\pi_{J_{k}}(Z)$ as a multinomial expansion in terms of $Z$-increments subordinated to the index set $J_{k}$.

If we first let $Z$ be a Lévy process, then we have a general method for constructing such increments. By adapting the construction described in Proposition 6.1, we define the random variables $\Delta_{m} \equiv \Delta_{J_{k}, m}(Z)$ by $Z_{t_{j m}}=Z_{t_{j_{m-1}}}+\Delta_{m}$ for $m \geq 2$, with $Z_{t_{j_{1}}}=Z_{t}+\Delta_{1}$ and $\Delta_{0}=Z_{t}$. The $\Delta_{m}$ then are independent random variables that can be regarded as being independent of the time- $t$ information $\mathcal{F}_{t}$ and as satisfying the identity in law

$$
\Delta_{m} \equiv \Delta_{J_{k}, m}(Z) \stackrel{\mathrm{D}}{=} Z_{\tau_{m}\left(J_{k}\right)},
$$

where $\tau_{m}\left(J_{k}\right)=t_{j_{m}}-t_{j_{m-1}}$ for $m \geq 2, \tau_{1}\left(J_{k}\right)=t_{j_{1}}-t$, and $\tau_{0}\left(J_{k}\right)=t$.

To study the effect of the variables $\Delta_{m}$ on $\pi_{J_{k}}(Z)$, represent any $\ell$ th factor of $\pi_{J_{k}}(Z)$ as the telescoping sum of the first $\ell+1$ of them. Picture the product $\pi_{J_{k}}(Z)$ as a triangular array of 
$k$ lines, corresponding to its factors, and $k+1$ columns, corresponding to the $\Delta_{m}$ each factor uses. The combinatorial problem to be solved for expanding this product is to characterize and enumerate the different $k$-tuples $L_{k}$ each of whose $m$ th entry, $\ell_{m}$, is obtained by choosing any element from the $m$ th row of the above array. To fix ideas, we have one $\Delta_{k}$ from the last row which either occurs or, respectively, does not occur in $L_{k}$ according to whether $\ell_{k}=1$ or $\ell_{k}=0$. We have two $\Delta_{k-1}$ s from the last and the next-to-last rows which cannot, however, both occur in $L_{k}$ if $\Delta_{k}$ already does. Thus, we have $\ell_{k-1} \leq 1+\left(1-\ell_{k}\right)$ occurrences of $\Delta_{k-1}$ which moreover arise by making $\ell_{k-1}$ choices from $1+\left(1-\ell_{k}\right)$ possibilities. Proceeding inductively, we thus arrive at the following result.

Proposition 7.1. If, in the above setting, $Z$ is a Lévy process, then we have the representation in law

$$
\pi_{J_{k}}(Z) \stackrel{\mathrm{D}}{=} \sum_{L_{k}} \Lambda\left(L_{k}\right) Z_{t}^{k-\left|L_{k}\right|} \prod_{m=1}^{k} \Delta_{m}^{\ell_{m}},
$$

where the sum is over all $k$-tuples $L_{k}=\left(\ell_{1}, \ldots, \ell_{k}\right)$ of nonnegative integers with length $\left|L_{k}\right|=\ell_{1}+\cdots+\ell_{k}$ which satisfy $\ell_{m} \leq \lambda(m)$. Here $\lambda \equiv \lambda_{L_{k}}$ is recursively defined by $\lambda(k)=1$ and $\lambda(k-m)=\lambda(k-(m-1))+1-\ell_{k-(m-1)}$ for any positive integer $m \leq k-1$, and the multiplicity factor $\Lambda\left(L_{k}\right)$ is given by the following product of binomial coefficients:

$$
\Lambda\left(L_{k}\right)=\prod_{m=1}^{k-1}\left(\begin{array}{c}
\lambda(m) \\
\ell_{m}
\end{array}\right) .
$$

This result affords us a representation of $R_{T}$ in terms of independent random variables, as desired. Its effect on stochastic functionals, however, depends on the maps $\rho$. The futures value case represents a case of maximal simplicity, in that $\rho$ is the identity map there, and is treated as follows.

Corollary 7.1. In the setting and notation of Proposition 7.1, we have the representation

$$
\mathrm{E}\left[\pi_{J_{k}}(Z) \mid \mathcal{F}_{t}\right]=\sum_{L_{k}} \Lambda\left(L_{k}\right) Z_{t}^{k-\left|L_{k}\right|} \prod_{m=1}^{k} \mathrm{E}\left[Z_{\tau_{m}\left(J_{k}\right)}^{\ell_{m}}\right] .
$$

Here both sides are well defined and finite if and only if, for any $\tau \equiv \tau_{m}\left(J_{k}\right)$, the moments $\mathrm{E}\left[Z_{\tau}^{a}\right]$ are well defined and finite for all nonnegative integers $a \leq k$.

When $Z$ is an exponential Lévy process we use the $\Delta$-construction as follows.

Proposition 7.2. If, in the above setting, $Z=\exp (X)$ for a Lévy process $X$, then we have the representation

$$
\pi_{J_{k}}(Z)=Z_{t}^{k} \prod_{\ell=1}^{k} Y_{\ell}^{k+1-\ell},
$$

where we define the random variables $Y_{\ell} \equiv Y_{J_{k}, \ell}(Z)$, for any positive integer $\ell \leq n$, by $Y_{\ell}=\exp \left(\Delta_{J_{k}, \ell}(X)\right)$.

Proof. On substitution of $Z_{t_{j_{\ell}}}=Z_{t} \prod_{m=1}^{\ell} Y_{m}$ into the defining relationship for $\pi_{J_{k}}$, we have $\pi_{J_{k}}(Z)=\prod_{\ell=1}^{k} Z_{t} \prod_{m=1}^{\ell} Y_{m}$. Then interchanging the order of the two products completes the proof of Proposition 7.2. 
The expectations of the $\pi_{J_{k}}(Z)$, as they are relevant for the futures case, will now be obtained in terms of the moment generating function of the exponentiated process.

Corollary 7.2. In the setting of Proposition 7.2, assume that the interval $[1, k]$ is contained in the domain of definition of the moment generating function, $M G_{X}$, of $X$. Then we have

$$
\mathrm{E}\left[\pi_{J_{k}}(Z) \mid \mathcal{F}_{t}\right]=Z_{t}^{k} \prod_{\ell=1}^{k} M G_{X}(k+1-\ell)^{\tau_{\ell}\left(J_{k}\right)} .
$$

To conclude this section, note how the explicit formulae in Corollaries 7.1 and 7.2 are expressed in terms of moment generating functions, or items derived from them such as the moments themselves. This is a characteristic feature of our approach to stochastic functionals as developed in the rest of the paper using Laguerre reduction series.

\section{Laguerre reduction series for discretely monitored stochastic functionals}

As the third main step in our approach, following on from Sections 5 and 6, in this section we develop Laguerre reduction series for discretely sampled stochastic functionals of positive random variables of the form

$$
\mathrm{E}[\rho(\Xi)], \quad \text { where } \Xi=\sum_{k=1}^{n} \gamma_{k} Y_{k}>0 .
$$

Here $\rho$ is any measurable function on the positive reals, $\gamma_{1}, \ldots, \gamma_{n}$ are any positive reals, and $Y_{1}, \ldots, Y_{n}$ are any independent random variables, which we specialize in three stages from this basic setting to the one envisaged in the normalization procedure of Section 6.

\subsection{Basic setting}

As a first subsidiary step, we specialize the two main results about Laguerre reduction series of Section 3 to the above situation. Using the concepts developed there, we first have the following immediate translation of Theorem 3.1.

Theorem 8.1. Denote by $g_{\Xi}$ the probability density function of $\Xi$, and let the real $\alpha>-1$ be such that $\rho$ and $g_{\Xi} / w_{\alpha}$ are both in $L_{\alpha}^{2}(0, \infty)$. Then we have the absolutely convergent Laguerre reduction series representation

$$
\mathrm{E}[\rho(\Xi)]=\sum_{m=0}^{\infty} a_{m}\left\langle\rho, L_{m}^{\alpha}\right\rangle_{\alpha},
$$

whose error terms, $R_{M}=\sum_{m=M}^{\infty} a_{m}\left\langle\rho, L_{m}^{\alpha}\right\rangle_{\alpha}$, satisfy the inequality

$$
R_{M}^{2} \leq\|\rho\|_{\alpha}^{2} \sum_{m=M}^{\infty} a_{m}^{2}\left\|L_{m}^{\alpha}\right\|_{\alpha}^{2}
$$

Recall from Section 3.1 the representation

$$
a_{m}=\sum_{k=0}^{m} \frac{\alpha_{m, k}}{\left\|L_{m}^{\alpha}\right\|_{\alpha}^{2}} \mathrm{E}\left[\Xi^{k}\right]
$$


which is in terms of the coefficients, $\alpha_{m, k}$, of $L_{m}^{\alpha}$ and the $k$ th moments, $\mathrm{E}\left[\Xi^{k}\right]$, of $\Xi$. For the latter recall from Proposition 2.1 the multinomial representation in terms of the moments of $Y_{\ell}$, given by

$$
\mathrm{E}\left[\Xi^{k}\right]=\sum_{|J|=k}\left(\begin{array}{l}
k \\
J
\end{array}\right) \gamma^{J} \prod_{\ell=1}^{n} \mathrm{E}\left[Y_{\ell}^{j_{\ell}}\right]
$$

where the sum is over all $n$-tuples $J=\left(j_{1}, \ldots, j_{n}\right)$ of nonnegative integers with $|J|=j_{1}+$ $\cdots+j_{n}=k$, and where $\gamma^{J}=\gamma_{1}^{j_{1}} \cdots \gamma_{n}^{j_{n}}$.

Note how the reduction series separates the stochastic side of the problem from the analytic side. The coefficients $a_{m}$ are expressed solely in terms of the moments of the $Y_{k}$, whereas the function $\rho$ enters by means of the inner product factors of the reduction series summands.

Sufficient conditions for the existence of the Theorem 8.1 Laguerre reduction series can be given in terms of data from the moment generating functions, $M G_{k}$, of any single $Y_{k}$. This requires concepts from Section 3.4 as well as the growth measure $\gamma_{\infty}$ of Section 3.2, and our precise result is as follows.

Theorem 8.2. In Theorem 8.1, in order that $g_{\Xi} / w_{\alpha}$ be in $L_{\alpha}^{2}(0, \infty)$ it is sufficient that the following two inequalities be satisfied:

$$
1<2 \min _{1 \leq k \leq n}\left\{\frac{\sigma_{\mathrm{sc}}\left(Y_{k}\right)}{\gamma_{k}}\right\}, \quad \alpha+3<2 \sum_{k=1}^{n} \gamma_{\infty}\left(\left|M G_{k}^{-}\right|\right) .
$$

This is a translation of Theorem 3.2 which in the present context requires that the two inequalities $\sigma_{\mathrm{sc}}(\Xi)>\frac{1}{2}$ and $\alpha+3<2 \gamma_{\infty}\left(\left|M G_{\Xi}^{-}\right|\right)$be satisfied. Here the moment generating function, $M G_{\Xi}$, of $\Xi$ is, by Proposition 6.3, the product of the $\gamma_{k}^{*}\left(M G_{k}\right)$. Its abscissa of convergence, $\sigma_{\mathrm{sc}}(\Xi):=\sup \{\operatorname{Re}(z): \mathrm{E}[\exp (z \Xi)]$ is finite $\}$, is thus the minimum of the corresponding abscissae of convergence $\sigma_{\mathrm{sc}}\left(\gamma_{k} Y_{k}\right)$. The relation $\gamma_{k} \sigma_{\mathrm{sc}}\left(\gamma_{k} Y_{k}\right)=\sigma_{\mathrm{sc}}\left(Y_{k}\right)$ can be checked to hold using the positivity of the reals $\gamma_{k}$. Similarly, the polynomial growth order at $\infty$ of $M G_{\Xi}^{-}(z):=M G_{\Xi}(-z)$ is the sum of the corresponding growth orders $\gamma_{\infty}\left(\left(\gamma_{k}^{*} M G_{k}\right)^{-}\right)$, and the latter can be checked to be equal to $\gamma_{\infty}\left(M G_{k}^{-}\right)$. This proves Theorem 8.2.

While the form of the Theorem 8.1 Laguerre reduction series remains unchanged, it is the expandability criterion of Theorem 8.2 which becomes sharper and more concrete upon specializing the $Y_{k}$. This we describe in the next two sections.

\subsection{Lévy process setting}

As a second subsidiary step, we specialize to discretely sampled functionals of positive Lévy processes $X$ as introduced in Section 4. Here the idea is first to apply to them the normalization procedure of Section 6. Recall that this entails changing not only the representing variables but also the representing functions as summarized in Proposition 6.1. The point is that, since we consider functionals of positive linear combinations of positive random variables, positivity of the representing variables is preserved as described in Addendum 6.1. This then puts us in the basic setting of Section 8.1 with the random variables $Y_{k}$ satisfying

$$
Y_{k} \stackrel{\mathrm{D}}{=} X_{\tau_{k}}
$$

for positive reals $\tau_{k}$.

The effect of this specialization on the Theorem 8.1 Laguerre reduction series is restricted to its coefficients $a_{m}$ : these are now expressed in terms of the moments of the $X_{\tau_{k}}$ by way of 
the moments of $\Xi$. Our sufficient conditions for this Laguerre reduction series to exist, on the other hand, become simpler and now just need the moment generating function, $M G_{X}$, of $X$. Our precise result is as follows.

Theorem 8.3. In the above Lévy process setting, sufficient for the absolute convergence of the Theorem 8.1 Laguerre reduction series for $\mathrm{E}[\rho(\Xi)]$ is that $\rho$ be in $L_{\alpha}^{2}(0, \infty)$ and that the following two inequalities be satisfied:

$$
\max _{1 \leq k \leq n}\left\{\gamma_{k}\right\}<2 \sigma_{\mathrm{sc}}\left(X_{1}\right), \quad \alpha+3<2(T-t) \gamma_{\infty}\left(\left|M G_{X}^{-}\right|\right)
$$

To see how this result is obtained by translating Theorem 8.2 to the current situation, first note that now the moment generating function, $M G_{k}$, of any $Y_{k}$ is the $\tau_{k}$ th power of the moment generating function, $M G_{X}$, of $X$. Hence, each abscissa of convergence $\sigma_{\mathrm{sc}}\left(Y_{k}\right)$ is equal to the abscissa of convergence $\sigma_{\mathrm{sc}}\left(X_{1}\right)$ of this last function, and the first inequality of the theorem thus follows on translating the first inequality of Theorem 8.2. To obtain the second inequality we use the fact that $\gamma_{\infty}$ is log-additive in the sense that raising its argument to the $a$ th power results in multiplication by $a$, i.e. $\gamma_{\infty}\left(f^{a}\right)=a \gamma_{\infty}(f)$. In the second inequality of Theorem 8.2 we thus have $\sum_{k=1}^{n} \gamma_{\infty}\left(\left|M G_{k}^{-}\right|\right)=\sum_{k=1}^{n} \tau_{k} \gamma_{\infty}\left(\left|M G_{X}^{-}\right|\right)$. Since the $\tau_{k}$ give rise to a telescoping sum, the second inequality of Theorem 8.3 follows.

\subsection{GIG Lévy process setting}

As a third subsidiary step, we now specialize the setting of Section 8.2 to the case in which $X$ is the GIG Lévy process associated with any triple $\xi=(\gamma, \delta, \lambda)$ in the sense of Section 2.2.

In the coefficients, $a_{m}$, in the Theorem 8.1 Laguerre reduction series for $\mathrm{E}[\rho(\Xi)]$, the moments of the $Y_{k}$ are now explicitly given by combining Propositions 2.1 and 2.2. Drastic simplifications occur in the conditions sufficient for these reduction series to converge. We state them as follows.

Theorem 8.4. In the above GIG Lévy process setting, sufficient for the absolute convergence of the Theorem 8.1 Laguerre reduction series for $\mathrm{E}[\rho(\Xi)]$ are the following two conditions: $\rho$ is in $L_{\alpha}^{2}(0, \infty)$ and $\max \left\{\gamma_{k}: 1 \leq k \leq n\right\}<\gamma^{2}$.

To see how this result is obtained by translating Theorem 8.3 to the present situation, first note from Section 2.2 that we have $\sigma_{\mathrm{sc}}\left(X_{1}\right)=\gamma^{2} / 2$ for the abscissa of convergence of the moment generating function of $X$. The first inequality of Theorem 8.3 thus translates into the inequality of Theorem 8.4. To analyze the second inequality required to be satisfied in Theorem 8.3, recall the asymptotic behaviour of $K$-Bessel functions: $K_{a}(z) \sim \sqrt{\pi /(2 z)} \exp (-z)$ as $|z|$ tends to $\infty$ in $\{\operatorname{Re}(z)>0\}$; see Lebedev (1972, Equation (5.11.9)). By inspection this implies the exponential decay to 0 of the moment generating function, $M G_{X}$, of $X$ as $|z|$ tends to $\infty$ in $\{\operatorname{Re}(z)<0\}$, since this function is a scalar multiple of a $K$-Bessel function. Hence, $\gamma_{\infty}\left(\left|M G_{X}^{-}\right|\right)=\infty$ as noted in Addendum 3.1. Thus, the second inequality of Theorem 8.3 is trivially true in the present situation, and it remains to require the $\alpha$-square integrability of $\rho$ in Theorem 8.3 also in Theorem 8.4. The proof of this result is then complete.

\section{Applications to Asian-type options}

To both illustrate and summarize our main results, in this section we study the explicit valuation of discretely sampled Asian-type options, discussed in Section 4, in certain Lévy process models. The results for such general options are given in Section 9.2, while in Section 9.3 we focus on Asian options proper. 


\subsection{General setting}

Let $X$ be any positive Lévy process as in Section 2.1, partition any subinterval $\left[t_{0}, T\right]$ of the nonnegative reals by the choice of $N$ times $t_{m}$ with $t_{1}<\cdots<t_{N}=T$, and consider the stochastic functionals given by the expectations

$$
H_{\Sigma, t}\left(\rho_{p, K}\right)=\mathrm{E}\left[\rho_{p, K}(\Sigma) \mid \mathcal{F}_{t}\right], \quad \text { where } \Sigma \equiv \Sigma_{N}^{a}=\sum_{m=1}^{N} a_{m} X_{t_{m}}
$$

Here the expectations are conditional on the information, $\mathcal{F}_{t}$, at any time $t$ in $\left[t_{0}, T\right]$; the functions $\rho_{p, K}$ are, for any integer $p \geq 1$ and any real $K>0$, given by

$$
\rho_{p, K}(y)=(y-K)_{+}^{p}
$$

on the reals, where $(x)_{+}=\max \{0, x\}$ for any real $x$; and the $a_{m}$ are any positive reals. We thus consider contingent claims on $\left[t_{0}, T\right]$ with time- $T$ payoff given by the $p$ th power of the excess over $K$ of $\Sigma$, the generalized average over time. We assume, moreover, that the valuation of these Asian-type options reduces to computing the functionals $H_{\Sigma, t}$. This will be the case in their risk-neutral valuation under deterministic interest rates, with $X$ modelling the price process of the underlying asset. Our argument is based on the normalization procedure of Section 6 , and is given in terms of the random variables $\Xi$ and their moments.

Summarizing Proposition 6.1, these random variables are the sums

$$
\Xi=\sum_{k=1}^{n} \gamma_{k} Y_{k}
$$

expressed in terms of the following quantities. Firstly, $n \equiv n_{t}$ denotes the number of summands in $\Sigma$ which are stochastic at time $t$, or, alternatively, $n=N-m_{t}$, where $m_{t}=\max \left\{m: t_{m} \leq t\right\}$ is the index of the last time, $t_{m}$, before or equal to $t$. Secondly, the $\gamma_{k}$ are the positive reals given by $\gamma_{k}=\sum_{\ell=k}^{n} a_{m_{t}+\ell}$. Thirdly, the $Y_{k}$ are independent random variables which are independent of time- $t$ information and determined in law by

$$
Y_{k} \stackrel{\mathrm{D}}{=} X_{\tau_{k}}
$$

where $\tau_{1}=t_{m_{t}+1}-t$ and $\tau_{k}=t_{m_{t}+k}-t_{m_{t}+k-1}$ for $2 \leq k \leq n$. From Addendum 6.1, note, moreover, that $\Xi$ is positive: $\Xi>0$.

As for the $M$ th moments of $\Xi$, refer to the recurrence rule of Proposition 6.4 and recall the multinomial expansion given there in terms of the moments of the $X_{\tau_{k}}$ by

$$
\mathrm{E}\left[\Xi^{M}\right]=\sum_{|J|=M}\left(\begin{array}{c}
M \\
J
\end{array}\right) \gamma^{J} \prod_{k=1}^{n} \mathrm{E}\left[X_{\tau_{k}}^{j_{k}}\right]
$$

where the sum is over all $n$-tuples $J=\left(j_{1}, \ldots, j_{n}\right)$ of nonnegative integers with $|J|=j_{1}+$ $\cdots+j_{n}=M$, and where $\gamma^{J}=\gamma_{1}^{j_{1}} \cdots \gamma_{n}^{j_{n}}$.

\subsection{Reduction series for Asian-type options}

Applying the concepts and notation of Section 9.1, in this section we develop a constructive structure theory for the functionals $H_{\Sigma, t}$ based on the Laguerre reduction series of Section 8. 
Here we observe a dichotomy in dependency on the sign of the parameter $q \equiv q_{t}$ defined as

$$
q=K-A_{t}, \quad \text { where } A_{t}=\sum_{m=1}^{N} a_{m} X_{t_{m} \wedge t} .
$$

With proofs deferred to the end of the section, our results can be summarized in two stages. If $q$ is nonpositive then the functionals $H_{\Sigma, t}$ are linear combinations of finitely many moments of the random variable $\Xi$ of Section 9.1 .

Theorem 9.1. If $q \leq 0$ then $H_{\Sigma, t}\left(\rho_{p, K}\right)=\sum_{m=1}^{p}(-q)^{p-m}\left(\begin{array}{c}p \\ m\end{array}\right) \mathrm{E}\left[\Xi^{m}\right]$.

If $q$ is positive then we represent the functionals $H_{\Sigma, t}$ by Laguerre reduction series.

Theorem 9.2. For $q>0$, let the reals $\alpha>-1$ and $c>0$ be such that the following two inequalities are satisfied:

$$
\frac{c}{q} \max _{1 \leq k \leq n}\left\{\gamma_{k}\right\}<2 \sigma_{\mathrm{sc}}\left(X_{1}\right), \quad \alpha+3<2\left(T-t_{0}\right) \gamma_{\infty}\left(\left|M G_{X}^{-}\right|\right) .
$$

Then we have the absolutely convergent Laguerre reduction series representation

$$
H_{\Sigma, t}\left(\rho_{p, K}\right)=\left(\frac{q}{c}\right)^{p} \sum_{m=0}^{\infty} a_{m}(c)\left\langle\rho_{p, c}, L_{m}^{\alpha}\right\rangle_{\alpha},
$$

whose error terms, $R_{M}=\sum_{m=M}^{\infty} a_{m}(c)\left\langle\rho_{p, c}, L_{m}^{\alpha}\right\rangle_{\alpha}$, satisfy

$$
R_{M}^{2} \leq\left\|\rho_{p, c}\right\|_{\alpha}^{2} \sum_{m=M}^{\infty} a_{m}(c)^{2}\left\|L_{m}^{\alpha}\right\|_{\alpha}^{2} .
$$

Refer to Section 3.4 for the definition of the abscissa of convergence, $\sigma_{\mathrm{sc}}\left(X_{1}\right)$, of $M G_{X}(z)=$ $\mathrm{E}\left[\exp \left(z X_{1}\right)\right]$, the moment generating function of $X$, and to Section 3.2 for the definition of the growth measure $\gamma_{\infty}$, which here is applied to the function $M G_{X}^{-}(z)=M G_{X}(-z)$. Of the other quantities introduced, the coefficients $a_{m}(c)$ are given by

$$
a_{m}(c)=\sum_{k=0}^{m} \frac{\alpha_{m, k}}{\left\|L_{m}^{\alpha}\right\|_{\alpha}^{2}}\left(\frac{c}{q}\right)^{k} \mathrm{E}\left[\Xi^{k}\right]
$$

in terms of the coefficients, $\alpha_{m, k}$, of the $m$ th $\alpha$-Laguerre polynomial, $L_{m}^{\alpha}$, as defined in Section 3.1 and the $k$ th moments, $\mathrm{E}\left[\Xi^{k}\right]$, of $\Xi$ as recalled in Section 9.1. Finally, we have

$$
\left\langle\rho_{p, c}, L_{m}^{\alpha}\right\rangle_{\alpha}=\sum_{k=0}^{m} \alpha_{m, k} \sum_{\ell=0}^{p}(-1)^{p} \frac{c^{p}}{c^{\ell}}\left(\begin{array}{l}
p \\
\ell
\end{array}\right) \gamma(\alpha+k+\ell+1, c)
$$

and

$$
\left\|\rho_{p, c}\right\|_{\alpha}^{2}=\sum_{\ell=0}^{2 p}(-1)^{\ell} \frac{c^{2 p}}{c^{\ell}}\left(\begin{array}{l}
p \\
\ell
\end{array}\right) \gamma(\alpha+\ell+1, c),
$$

in terms of the (lower) incomplete gamma function, given by $\gamma(s, x)=\int_{0}^{x} u^{s-1} \exp (-u) \mathrm{d} u$ for any positive reals $s$ and $x$; see Erdélyi et al. (1981, Chapter IX) for more details of this function.

Specializing $X$ to any GIG Lévy process, we have the following consequence of this last result. 
Corollary 9.1. Let $X$ be the GIG Lévy process associated with a triple $\xi=(\gamma, \delta, \lambda)$ as described in Section 2.2. Then validity of the inequality $0<2(c / q) \max \left\{\gamma_{k}: 1 \leq k \leq n\right\}<\gamma^{2}$ is sufficient for the absolute convergence of the Laguerre reduction series of Theorem 9.2.

Deferring the proofs briefly, note in this situation the recurrence rule and the explicit formulae for the moments of $X$ in Section 2.2. For any positive integer $M$, they assert that

$$
\mathrm{E}\left[X_{\tau}^{M}\right]=\sum_{m=1}^{M}(-1)^{m}(-\tau)_{m} \sum_{\ell=0}^{m-1} \frac{(-1)^{m}}{(m-\ell) ! \ell !} F_{m-\ell}^{M}(0),
$$

where

$$
F_{M}^{m}(0)=\sum_{|L|=M}\left(\begin{array}{l}
n \\
L
\end{array}\right) \prod_{j=1}^{m}\left(\frac{\delta}{\gamma}\right)^{\ell_{j}} \frac{K_{\lambda+\ell_{j}}(\gamma \delta)}{K_{\lambda}(\gamma \delta)}
$$

with the sum running over all $m$-tuples $L=\left(\ell_{1}, \ldots, \ell_{m}\right)$ of nonnegative integers such that $|L|=\ell_{1}+\cdots+\ell_{m}=M$, and with $K_{a}$ the $K$-Bessel function of any complex order $a$; see Lebedev (1972, Chapter 5) for more details of these functions.

Proof of Theorems 9.1 and 9.2 and Corollary 9.1. The starting point for this section's results is the identity

$$
H_{\Sigma, t}\left(\rho_{p, K}\right)=\mathrm{E}\left[\rho_{p, q}(\Xi)\right]
$$

On recalling that $q=K-A_{t}$, this simply gives expression to the normalization procedure of Section 6 as summarized by Proposition 6.1, and Theorem 9.1 follows immediately.

Thus, assuming that $q>0$, note that this section's framework places us variously in the general Lévy process setting of Section 8.2 and the GIG Lévy process setting of Section 8.3. We prove the remaining two results by translating the pertinent results of those sections. Temporarily writing $\Xi_{a}=a \Xi$ for any positive real $a$, the proofs are now based on the identity

$$
\mathrm{E}\left[\rho_{p, q}(\Xi)\right]=(q / c)^{p} \mathrm{E}\left[\rho_{p, c}\left(\Xi_{c / q}\right)\right]
$$

which follows directly from the homogeneity properties of $\rho$. The principal idea of using this identity is to obtain expansions into Laguerre reduction series by applying Theorem 8.1 to the right-hand side and then to use the sufficiency criteria of Theorem 8.3 or Theorem 8.4 (as appropriate to the type of process considered) to ensure convergence.

First considering the general Lévy process case, grant for a moment that the assumptions of Theorem 9.2 imply satisfaction of the sufficient conditions expressed by the two inequalities of Theorem 8.3. Then, applying Theorem 8.1 with $\rho=\rho_{p, c}$ and $\Xi=\Xi_{c / q}$, the Theorem 9.2 reduction series follows immediately, and direct computations moreover establish the explicit formulae for the pertinent values of the bilinear form. To reduce the two inequalities of Theorem 8.3 to those of Theorem 9.2, first note that the respective second inequalities are identical. The first inequality of Theorem 8.3, on the other hand, requires that $\max \left\{\gamma_{k}^{*}\right\}<2 \sigma_{\mathrm{sc}}\left(X_{1}\right)$, with $\gamma_{k}^{*}$ the coefficients of $\Xi_{c / q}$. These are the products of $c / q$ and the respective coefficients, $\gamma_{k}$, of $\Xi$, and the proof of Theorem 9.2 is complete.

In the GIG Lévy process case, a similar translation of the inequalities of Theorem 8.4 establishes the assertions of Corollary 9.1. The proof of this section's results is thus complete. 


\subsection{Reduction series for Asian options}

In this section we spell out the implications of the Section 9.2 results for the risk-neutral valuation of discretely monitored Asian options. Assuming there to be a constant short rate $r>0$, we focus on the functionals $C_{t}(K)$ given by

$$
C_{t}(K)=\exp (-r(T-t)) H_{\Sigma, t}\left(\rho_{p, K}\right)
$$

where

$$
H_{\Sigma, t}\left(\rho_{p, K}\right)=\mathrm{E}\left[\rho_{p, K}\left(\frac{1}{N} \sum_{m=1}^{N} X_{t_{m}}\right) \mid \mathcal{F}_{t}\right] .
$$

Working under any equivalent martingale measure as indicated in Section 9.1, these represent the time- $t$ values of a certain contingent claim, namely the Asian $p$-power call, discretely sampled at finitely many time points, $t_{1}, \ldots, t_{N}$, of its monitoring period $\left[t_{0}, T\right]$, written on an asset with price process $X$ and strike price $K$.

In the setting of Section 9.1, this corresponds to taking equal weights $a=\left(a_{1}, \ldots, a_{N}\right)$, where $a_{m}=1 / N$ for all $m$. The parameter $q \equiv q_{t}$, which determines the structure of the functionals $H_{\Sigma, t}$, is thus given by

$$
q=K-\frac{1}{N} \sum_{m=1}^{N} X_{t_{m} \wedge t}
$$

and the normalized coefficients $\gamma_{k}$ become $\gamma_{k}=(1 / N)\left(n_{t}-k+1\right)$ for $1 \leq k \leq n_{t}$.

This entails simplifications in the sufficient conditions developed in Section 9.2 for the convergence of the Laguerre reduction series for the functionals $H_{\Sigma, t}$. First, for general Lévy processes $X$, we have the following specialization of Theorem 9.2.

Theorem 9.3. Let $q>0$. Then the Theorem 9.2 Laguerre reduction series for $H_{\Sigma, t}\left(\rho_{p, K}\right)$, for any reals $\alpha>-1$ and $c>0$, is absolutely convergent if the following two inequalities are satisfied:

$$
\frac{c}{q} \frac{n_{t}}{N}<2 \sigma_{\mathrm{sc}}\left(X_{1}\right), \quad \alpha+3<2(T-t) \gamma_{\infty}\left(\left|M G_{X}^{-}\right|\right)
$$

Second, specializing $X$ to be any GIG Lévy process as in Section 2.2, Corollary 9.1 translates to the following result.

Theorem 9.4. Let $X$ be the GIG Lévy process associated with any triple $\xi=(\gamma, \delta, \lambda)$ as described in Section 2.2, and assume that $q>0$. Then the Theorem 9.2 Laguerre reduction series for $H_{\Sigma, t}\left(\rho_{p, K}\right)$, for any reals $\alpha>-1$ and $c>0$, is absolutely convergent if the following single inequality is satisfied: $c / q<\left(N / n_{t}\right) \gamma^{2}$.

\subsection{Complements}

To conclude, in particular, the discussion in this section of Asian option valuation, it remains to address two issues of practical relevance: the computation and the construction of the functionals $H_{\Sigma, t}$. 
First we address computation with the reduction series. On having chosen any equivalent martingale measure, this principally proceeds along the lines developed in Schröder (2005a, Sections 9.2 and 9.3) and Schröder (2006b, Section 8.2) for valuing continuously sampled Asian options in the Black-Scholes model, as follows. As a first step, compute the number of moments of $\Sigma$ to be worked with as indicated in Section 9.1. As a second step, then use the Theorem 9.2 error estimate to determine 'maximal accuracies' of the reduction series by way of determining 'good choices' for the parameters $\alpha$ and $c$. As a third and final step, sum the resulting reduction series. The explicit determination of equivalent martingale measures is given ample space in the theses of Prause (1999) and Raible (2000), as summarized in Eberlein (2001) and Nicolato and Venardos (2003). The empirical work reported in these references moreover provides realistic size ranges for the model parameters. These we have used in preliminary computations which seemed to indicate that the convergence behaviour of the present paper's reduction series resembles the one observed in Schröder (2005a), (2006b) in qualitative and absolute terms. The author hopes to be able to consider these issues in future.

To now address construction of the functionals, Schröder (2006a) has stressed for this the importance of ladder height reduction series. Originating with Dufresne (2000), this concept was in fact developed with properties and usages complementary to those of the present paper's reduction series. To fix ideas about a typical application, and to indicate the potential of this notion, Schröder (2006a, Section 6.4), for example, in the present setting, obtained a principal method for explicitly determining risk-neutral parameters for Asian option valuation using the prices of 'plain-vanilla' options quoted on the markets.

\section{Epilogue}

The principal validity of the present paper's approach goes beyond the class of stochastic functionals we have concentrated on up to now. To conclude the paper, we turn from our main focus to reconsider from Section 4 the discretely sampled stochastic functionals in $N$ positive random variables, i.e.

$$
\mathrm{E}\left[R\left(\boldsymbol{X}_{t}\right)\right]
$$

We will use multi-index notation to stress the analogy with the one-dimensional case. Thus, we have a vector $\boldsymbol{t}=\left(t_{1}, \ldots, t_{N}\right)$ of sample times and a vector $\boldsymbol{X}_{\boldsymbol{t}}=\left(X_{t_{1}}, \ldots, X_{t_{N}}\right)$ of positive random variables $X_{t_{m}}$. Proceeding along the lines indicated in Section 6, to begin with we now consider the functionals not as functionals of the process $X$ but as functionals of the increments of $X$ :

$$
\mathrm{E}\left[R\left(\boldsymbol{X}_{\boldsymbol{t}}\right)\right]=\mathrm{E}\left[\lambda^{*}(R)\left(\Delta_{t}(\boldsymbol{X})\right)\right]
$$

Here $\Delta_{t}(\boldsymbol{X})=\left(\Delta_{1} X, \ldots, \Delta_{N} X\right)$ is the vector of increments $\Delta_{m} X=X_{t_{m}}-X_{t_{m-1}}$, where $X_{t_{0}}=x_{0}$ is any positive real, and $\lambda^{*}(R)=R \circ \lambda$, where $\lambda=\left(\lambda_{1}, \ldots, \lambda_{N}\right)$ with the maps $\lambda_{m}$ given by $\lambda_{m}\left(x_{1}, \ldots, x_{N}\right)=x_{0}+\sum_{\ell=1}^{m} x_{\ell}$.

Next we seek Laguerre reduction series for these functionals, and in analogy with the results of Sections 3 and 8 the result ought to be the absolutely convergent series

$$
\mathrm{E}\left[\lambda^{*}(R)\left(\Delta_{t}(\boldsymbol{X})\right)\right]=\sum_{J}\left\langle R, \mathcal{L}_{J}^{\alpha}\right\rangle_{\alpha} \frac{1}{\left\|\mathcal{L}_{J}^{\alpha}\right\|_{\alpha}} \mathrm{E}\left[\mathcal{L}_{J}^{\alpha}\left(\Delta_{t}(\boldsymbol{X})\right)\right]
$$

To make sense of this expression, the idea is to apply consecutively the results about singlevariable Laguerre reduction series of Sections 3 and 8. As a first step, thus consider its lefthand side to be a functional of $\Delta_{N} X$ alone and represent this partial functional by its Laguerre 
reduction series. The independence of the increments $\Delta_{m} X$ then entails that the effect of $\Delta_{N} X$ on the coefficients of this series is averaged out by the respective integrations. Each of them thus becomes a functional of the first $N-1$ increments, $\Delta_{1} X, \ldots, \Delta_{N-1} X$. Now iterate the construction with each of these coefficients. As it is the result of this construction process taken over $N$ steps, the above sum extends over all $N$-tuples $J=\left(j_{1}, \ldots, j_{N}\right)$ of nonnegative integers and represents an element of the space $L_{\alpha}^{2}\left(\left(\mathbb{R}_{>0}\right)^{N}\right)$, where $\alpha=\left(\alpha_{1}, \ldots, \alpha_{N}\right)$ for reals $\alpha_{m}>-1$; see Thangavelu (1993, Chapter 6). These spaces are obvious extensions of the one-dimensional ones recalled in Section 3.1. Thus, they carry the bilinear form

$$
\langle f, g\rangle_{\alpha}=\int_{\left(\mathbb{R}_{>0}\right)^{N}} w_{\alpha}(x) f(x) g(x) \mathrm{d} x,
$$

where

$$
w_{\alpha}\left(x_{1}, \ldots, x_{N}\right)=\prod_{m=1}^{N} w_{\alpha_{m}}\left(x_{m}\right),
$$

with respect to which the polynomials $\mathcal{L}_{J}^{\alpha}=\prod_{m=1}^{N} L_{j_{m}}^{\alpha_{m}}$ furnish an orthogonal basis. Conditions analogous to those of Theorems 3.1 and 8.3 (as appropriate) then do indeed guarantee the absolute convergence of the above series.

On the probabilistic side, computing these functionals is thus reduced to computing the multimoments of $\Delta_{t}(X)$ implicit in $\mathrm{E}\left[\mathcal{L}_{J}^{\alpha}\left(\Delta_{t}(\boldsymbol{X})\right)\right]$, and thus to computing the moments of any single $\Delta_{m} X$. On the analytic side, however, the coefficients $\left\langle R, \mathcal{L}_{J}^{\alpha}\right\rangle_{\alpha}$ are expressed in terms of $N$-dimensional integrals. Tractability of the problem from the stochastic point of view thus has a price from the analytic point of view.

This should in fact be taken as justifying our strategy as outlined in Section 4: put restrictions on $R$ that ensure the analytic tractability of the approach while still being general enough to permit meaningful applications. The factorization scheme outlined in Section 4 provides a systematic way of developing such specializations and permits one to address quite a broad range of problems. In Schröder (2005b), for example, this paper's results were extended from positive Lévy processes to exponential Lévy processes. We would therefore be more than gratified if the present paper was to initiate further work in this area connecting analysis, stochastics, and their applications.

\section{Acknowledgements}

I wish to thank Professor Eberlein (Universität Freiburg) for having brought Lévy processes and their applications to my attention, and for his continued interest in my work. I am grateful to the Mathematics Department of ETH, Zürich, for their hospitality and support during the writing of this paper. While the usual disclaimer applies, I wish to thank Dr A. Ostaszewski (London School of Economics) for a constructive and careful reading of a preliminary version of the paper, thanks I would like to extend to the referee as well.

\section{References}

Aase Nielsen, J. and Sandmann, K. (1995). Equity linked life insurance. Insurance Math. Econom. 16, $225-253$.

Aase Nielsen, J. AND SANDMAnN, K. (1996). Uniqueness of the fair premium for equity-linked life insurance contracts. Geneva Papers Risk Insurance Theory 21, 65-102.

Aase Nielsen, J. And Sandmann, K. (2002). The fair premium of an equity-linked life and pension insurance. In Advances in Finance and Stochastics, eds P. Schönbucher and K. Sandmann, Springer, Heidelberg, pp. 218-255.

BARndorfF-Nielsen, O. E. ANd Shephard, N. (2001). Modelling by Lévy processes for financial econometrics. In Lévy Processes, Birkhäuser, Boston, MA, pp. 283-318. 
Bauer, H. (1996). Probability Theory. De Gruyter, Berlin.

Doetsch, G. (1971). Handbuch der Laplacetransformation I. Birkhäuser, Basel.

Duffie, D. (1996). Dynamic Asset Pricing Theory, 2nd edn. Princeton University Press.

Dufresne, D. (2000). Laguerre series for Asian and other options. Math. Finance 10, 407-428.

Eberlein, E. (2001). Application of generalized hyperbolic Lévy motions to finance. In Lévy Processes, Birkhäuser, Boston, MA, pp. 319-336.

ERDÉLYI, A. et al. (1981). Higher Transcendental Functions, Vol. II. Krieger, Malabar, FL.

Hämmerlin, G. And Hoffmann, K. H. (1989). Numerische Mathematik. Springer, Heidelberg.

Hull, J. C. And White, A. (1987). The pricing of options on assets with stochastic volatilities. J. Finance 42, 281-300.

JøRgensen, B. (1982). Statistical Properties of the Generalized Inverse Gaussian Distribution. Springer, Heidelberg.

Kallenberg, O. (2002). Foundations of Modern Probability, 2nd edn. Springer, New York.

Lebedev, N. N. (1972). Special Functions and Their Applications. Dover, New York.

LIFFE (2004). Exchange contract no. 59. One month Euro overnight index average ("EONIA") indexed contract. The London International Financial Futures and Options Exchange. Available at http://www.euronext.com/ trader/contractspecifications/derivative/wide/0,5786,1732_627725,00.html?euronextCode=EON-LON-FUT.

Musiela, M. And RutKowski, M. (1997). Martingale Methods in Financial Modelling. Springer, New York.

Nicolato, E. And Venardos, E. (2003). Option pricing in stochastic volatility models of the Ornstein-Uhlenbeck type. Math. Finance 13, 445-466.

Prause, K. (1999). The generalized hyperbolic model. Doctoral Thesis, Universität Freiburg.

RAIBLE, S. (2000). Lévy processes in finance. Doctoral Thesis, Universität Freiburg.

Sansone, G. (1991). Orthogonal Functions. Dover, New York.

SCHRÖDER, M. (2005a). Laguerre series in contingent claim valuation, with applications to Asian options. Math. Finance 15, 491-531.

SCHRÖDER, M. (2005b). Continuous time methods in the study of discretely sampled functionals of Lévy processes, II: the case of exponential Lévy processes. Working paper.

SchröDER, M. (2005c). Continuous time methods in the study of discretely sampled functionals of Lévy processes, III: stochastic volatility models of OU type. Working paper.

SCHRöDER, M. (2006a). On ladder height densities and Laguerre series in the study of stochastic functionals. I. Basic methods and results. Adv. Appl. Prob. 38, 969-994.

SchröDER, M. (2006b). On ladder height densities and Laguerre series in the study of stochastic functionals. II. Exponential functionals of Brownian motion and Asian option values. Adv. Appl. Prob. 38, 995-1027.

Thangavelu, S. (1993). Lectures on Hermite and Laguerre Expansions. Princeton University Press. 